\title{
Photonic Crystal Fiber Interferometer for Dew Detection
}

\author{
Jinesh Mathew \\ Technological University Dublin \\ Yuliya Semenova \\ Technological University Dublin, yuliya.semenova@tudublin.ie \\ Gerald Farrell \\ Technological University Dublin, gerald.farrell@tudublin.ie
}

Follow this and additional works at: https://arrow.tudublin.ie/prcart

Part of the Optics Commons

\section{Recommended Citation}

Mathew, J., Semenova, Y. \& Farrell, G.: Photonic Crystal Fiber Interferometer for Dew Detection. Journal of Lightwave Technology, Vol. 30, no.8, 15 April, pp.1150-1155. doi:10.1109/JLT.2011.2170815

This Article is brought to you for free and open access by the Photonics Research Centre at ARROW@TU Dublin. It has been accepted for inclusion in Articles by an authorized administrator of ARROW@TU Dublin. For more information, please contact arrow.admin@tudublin.ie, aisling.coyne@tudublin.ie,gerard.connolly@tudublin.ie.

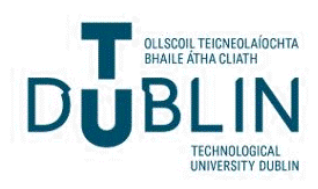




\title{
Photonic Crystal Fiber Interferometer for Dew Detection
}

\author{
Jinesh Mathew, Yuliya Semenova, and Gerald Farrell
}

(Invited Paper)

\begin{abstract}
A novel method for dew detection based on photonic crystal fiber (PCF) interferometer that operates in reflection mode is presented in this paper. The fabrication of the sensor head is simple since it only involves cleaving and fusion splicing. The sensor shows good sensitivity to dew formation with a large wavelength peak shift of the interference pattern at the onset of dew formation. The device's response to ambient humidity and temperature are also studied and reported in this paper. From our experiment it is also concluded that by attaching a thermoelectric cooler with temperature feedback, the sensor head demonstrated can be used as a miniature dew point hygrometer.
\end{abstract}

Index Terms-Dew sensor, humidity measurement, modal interferometer, optical fiber devices, photonics crystal fiber.

\section{INTRODUCTION}

$\mathbf{H}$ UMIDITY refers to the water vapor content in air or other gases. Humidity measurements can be stated in a variety of terms and units. The three commonly used terms are absolute humidity, relative humidity $(\mathrm{RH})$ and dew point. Absolute humidity is the ratio of the mass of water vapor to the volume of air or gas. It is commonly expressed in grams per cubic meter. The ratio of the percentage of water vapor present in air at a particular temperature and pressure to the maximum amount of water vapor the air can hold at that temperature and pressure is the relative humidity. Dew point, expressed in ${ }^{\circ} \mathrm{C}$ or ${ }^{\circ} \mathrm{F}$, is the temperature and pressure at which a gas begins to condense into a liquid. Dew point is a function of the pressure of the gas but it is independent of temperature and is therefore defined as fundamental. Dew (condensed moisture) is a problem in the fields of precision electrical devices, automobiles, air conditioning systems, warehouses and domestic equipment, etc. High humidity and condensation can create an environment where the development of mould on the wooden parts can take place and it can also cause corrosion of iron parts. This is a major problem in the case of the works of art in the museums and churches [1]. So there is a strong demand for a sensor able to accurately detect a high humidity or dew condensation state.

Manuscript received June 30, 2011; revised August 16, 2011; accepted September 28, 2011. Date of publication October 10, 2011; date of current version March 14, 2012.

The authors are with Photonics Research Center, Dublin Institute of Technology, Dublin, Ireland (e-mail: jinesh.mathew@mydit.ie; yuliya.semenova@dit.ie; gerald.farrell@dit.ie).

Color versions of one or more of the figures in this paper are available online at http://ieeexplore.ieee.org.

Digital Object Identifier 10.1109/JLT.2011.2170815
Different dew point hygrometers reported to date are based on resistive [2], capacitive [3], gravimetric [4], surface acoustic wave [5] or optical [6]-[10] technologies. Optical fibers are used to launch and collect optical signal from the condensation surface of chilled mirror hygrometers [9], [10]. Optical fiber dew sensors offer specific advantages, such as small size and weight, immunity to electromagnetic interference, corrosion resistance and remote operation, by comparison to their conventional electronic counterparts. Approaches to dew detection using optical fiber have been previously reported in [11], [12]. The working principle of these sensors is based on the change in the reflectivity which is observed on the surface of the fiber tip, when a water layer is formed on its distal end. The dependence on reflected power measurement scheme used in [11], [12] increases the chance of measurement error due to source power fluctuations. Recently we have demonstrated a simple sensor head for dew detection based on a photonic crystal fiber interferometer (PCFI) operated in reflection mode [13], with the advantage of good dew point measurement accuracy. The fabrication of such a sensor is very simple since it only involves cleaving and fusion splicing. Furthermore, the spectral measurement technique utilized in this work is free from errors due to source power variations. In this paper we report a detailed study of such a sensor for dew detection, including a study of the humidity and temperature dependence of the device with different lengths of PCF. Since the sensor head is fabricated from a single material, silica, its temperature dependence is very low. We investigate in detail the dew sensor performance with different lengths of PCF and it is found that a device with a compact length of PCF is suitable for dew sensing albeit with a reduction in the speed of response. The response of the sensor at different ambient humidity values is also investigated and reported in this paper.

\section{DeW Sensing Principle}

To study the response of the PCFI to dew formation it is required to set the temperature of the PCFI to dew point temperature, which is obtained from the values of ambient relative humidity and temperature. To do this consider a quantity of air with a constant water vapor concentration at a certain temperature, $\mathrm{T}$ and relative humidity, $\mathrm{RH}<100 \%$. The dew point temperature $\mathrm{T}_{\mathrm{d}}$ is defined as the temperature to which this quantity of air must be cooled down such that, at a constant pressure, condensation occurs $(\mathrm{RH}=100 \%)$. In terms of relative humidity $\mathrm{RH}$ and temperature $\mathrm{T}$, the dew point temperature is given as: 


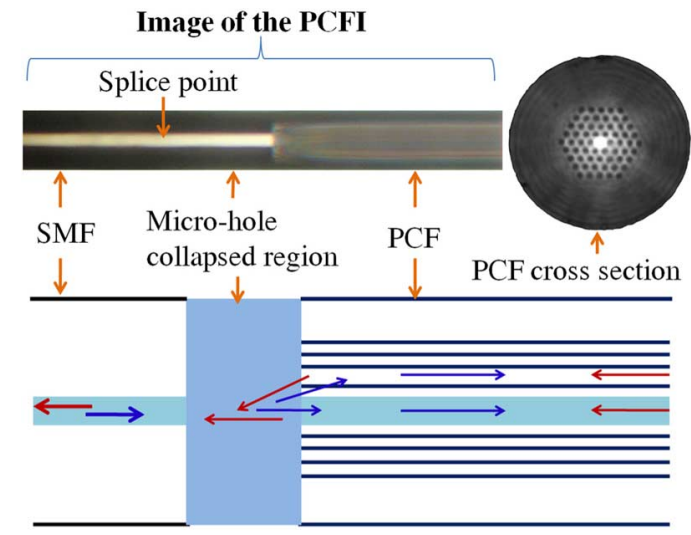

Excitation/recombination of modes

Fig. 1. Microscope image of the PCFI (upper) \& a schematic of the excitation/ recombination of modes in the hole collapsed region (lower).

$$
\mathrm{T}_{\mathrm{d}}(T, \mathrm{RH})=\alpha \frac{\ln \left(\frac{\mathrm{RH}}{100 \%}\right)+\frac{\beta T}{\alpha+T}}{\beta-\ln \left(\frac{\mathrm{RH}}{100 \%}\right)-\frac{\beta T}{\alpha+T}}
$$

where, $\alpha=243.12^{\circ} \mathrm{C}$ and $\beta=17.62$ are the so-called Magnus parameters for the temperature range -45 to $60^{\circ} \mathrm{C}$.

Photonic crystal fiber interferometers have attained great importance in recent times due to the simple fabrication process involved and excellent sensing performance [14]-[22]. In a PCFI the excitation and recombination of modes can be carried out by the hole collapsed region of the PCF [14], [18]. A microscopic image of the PCFI and a schematic of the excitation and recombination of modes in the PCFI are shown in Fig. 1. The fundamental SMF mode begins to diffract when it enters the collapsed section of the PCF. Because of diffraction, the mode broadens; depending on the modal characteristics of the PCF and the hole collapsed region, the power in the input beam can be coupled to the fundamental core mode and to higher order core modes [14]-[16] or to cladding modes [18]-[21] of the PCF. The modes propagate through the PCF until they reach the cleaved end from where they are reflected. Since the modes propagate at different phase velocities, thus in a certain length of PCF the modes accumulate a differential phase shift. Therefore, constructive or destructive interference occurs along the length of PCF. The phase velocities and phase difference are also wavelength dependent; therefore the optical power reflected by the device will be a maximum at certain wavelengths and minimum at others [17]. When the reflected modes re-enter the collapsed region they will further diffract and because the mode field of the SMF is smaller, the core acts as a spatial filter and picks up only a part of the resultant intensity distribution of the interference pattern in the PCF. A regular interference pattern in the reflection spectrum of the PCFI suggests that only two modes are interfering in the device. In our reported work [22] on a PCFI using LMA 10 fiber, based on the fact that higher order modes can exist in the core of a PCF with a short length [23], [24], we considered the interfering modes in the PCF to be two core modes. However in a later experiment, which involved varying the refractive index surrounding the cladding of a PCFI, we have observed good ambient refractive index sensitivity for a
PCFI fabricated using the same LMA 10 fiber. This suggests that the interfering modes are a core mode and a cladding mode of the PCF, a conclusion that is supported by [18], [21] for an LMA10 fiber. Thus, considering a core mode and a cladding mode as the interfering modes of the PCFI and designating the effective refractive indexes of the core mode as $n_{c}$ and cladding mode as $n_{\mathrm{cl}}$, the accumulated phase difference is $2 \pi \Delta n(2 L) / \lambda$, where $\Delta n=n_{c}-n_{\mathrm{cl}}, \lambda$ the wavelength of the optical source, and $L$ the physical length of the PCFI [15]. The power reflection spectrum of this interferometer will be proportional to $\cos (4 \pi \Delta n L / \lambda)$. The wavelengths at which the reflection spectrum shows maxima are those that satisfy the condition $4 \pi \Delta n L / \lambda=2 m \pi$, with $m$ being an integer [15]. This means that a periodic constructive interference occurs when $\lambda m=(2 \Delta n L / m)$. If some external stimulus changes $\Delta n$ (while $L$ is fixed) the position of each interference peak will change, a principle which allows the device to be used for sensing.

We have recently reported the relative humidity response of a PCFI [22]. An increase in humidity causes the shift of the interference pattern of a PCFI toward longer wavelengths and the value of this interference peak shift is exponential with respect to relative humidity. We attribute the shift of the interference peak to the adsorption and desorption of $\mathrm{H}_{2} \mathrm{O}$ molecules along the surface of holes within the PCF, at the interface between air and silica glass. Since the whole device is exposed to humidity the adsorption and desorption of water vapor on the PCF outer surface and on the end face also contribute to the shift of the interference pattern. But considering the field distribution of the interfering cladding mode shown in [21], [24] and below the dew point temperature the main contribution to the interference shift is considered to be due to the adsorption of water molecules within the voids of the PCF. The adsorption on the end face mainly causes a shift in overall power level of the interference pattern. Evolution of an adsorbed water layer structure on silicon oxide at room temperature has been demonstrated in [25]. Tiefenthaler and Lukosz [26] have shown that adsorption and desorption of water vapor by the surface of a waveguide changes the effective refractive index (RI) of the guided modes, in their case for a humidity sensor based on an integrated optical grating coupler. In the case of a PCFI a similar adsorption and desorption of water vapor changes the effective refractive index $\left(n_{\mathrm{cl}}\right)$ of the interfering cladding mode propagating in the PCF.

In [25] it was shown from the adsorption isotherm that the thickness of the adsorbed layer on a hydrophilic silicon oxide surface at room temperature starts increasing exponentially above $60 \% \mathrm{RH}$. The effective RI of the interfering cladding mode changes with respect to the thickness of this adsorbed layer. If we decrease the temperature of the PCFI the relative humidity close to the PCFI increases. As we further decrease the temperature of the PCFI the relative humidity becomes $100 \%$ or reaches the dew point temperature and hence the water vapor starts to condense. The condensed water vapor on the PCF causes a large phase change and therefore a large wavelength shift of the interference peaks is expected.

\section{EXPERIMENTAL INVESTIGATION}

The sensor system is composed of a broadband light source (SLED), a fiber coupler/circulator (FOC), the PCF interferom- 


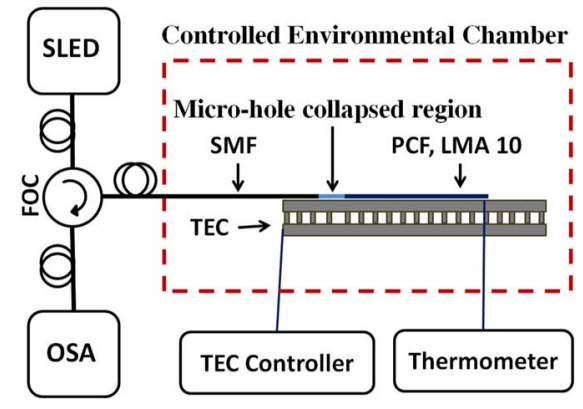

Fig. 2. Experimental setup to study the response of the PCFI to dew formation.

eter or sensor head, and an optical spectrum analyser (OSA) as shown in Fig. 2. The sensor head, as the main part of the sensor system, is composed of a small stub of PCF fusion spliced to the end of a standard SMF. The PCF in the sensor head has a microhole collapsed region near the splicing point and the free end of the PCF is exposed to ambient air.

Fusion splicing of the PCF to the SMF is undertaken using the electric arc discharge of a conventional arc fusion splicer. During the splicing process the voids of the PCF collapse through surface tension within a microscopic region close to the splice point. In fabricating such an interferometer, one critical condition for good sensor performance is achieving a regular interference pattern and good interference fringe visibility. The visibility of the interferometer depends on the power in the excited modes, which in turn depends on the length of the collapsed region [16]. However a long collapsed region length causes activation of many cladding modes and therefore degrades the sinusoidal nature of the interference patterns and furthermore increases the splice loss. Therefore, for an improved sensor performance, only one cladding mode is preferred due to its simple interference with the core mode. The collapsed region length can be controlled by the arc power and duration [16]. In our experiment, PCF (LMA10, NKT Photonics) designed for an endless single-mode operation was used. It has four layers of air holes arranged in a hexagonal pattern around a solid silica core. The light guidance mechanism in such a fiber is by means of modified total internal reflection. The dimensions of the LMA-10 PCF simplify alignment and splicing with the SMF with a standard splicing machine and minimize the loss due to mode field diameter mismatch compared to other PCFs. For the interferometer fabricated in this study the total length of the collapsed region is $200 \mu \mathrm{m}$. After fusion splicing, the PCF is cleaved using a standard fiber cleaving machine so that the end surface of the PCF acts as a reflecting surface.

Initially to investigate the influence the length of the PCFI on the fringe spacing a range thirteen PCFIs were fabricated with lengths from $3.5 \mathrm{~mm}$ to circa $100 \mathrm{~mm}$ long. As an example Fig. 3 shows the measured reflection spectra of three PCFIs in the $1500-1600 \mathrm{~nm}$ range with lengths of $92,10.5$ and $3.5 \mathrm{~mm}$. The reflection spectra of the interferometers exhibit regular interference patterns with a period or fringe spacing inversely proportional to the length of the PCF section. A modulation of the expected sinusoidal pattern is observed for the interference pattern shown in Fig. 3, which might be due to the excitation of more than one cladding mode or possibly due to the polarization

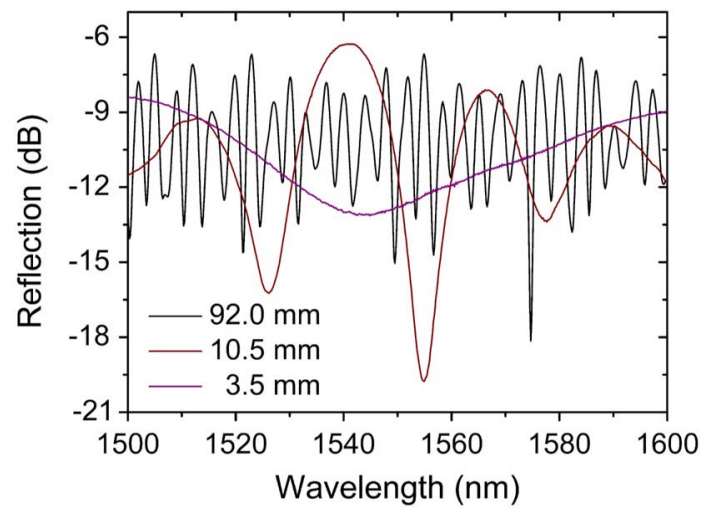

Fig. 3. The reflection spectra of interferometers with $\mathrm{L}=92 \mathrm{~mm}, 10.5 \mathrm{~mm}$ and $3.5 \mathrm{~mm}$ in the wavelength range of $1500-1600 \mathrm{~nm}$.

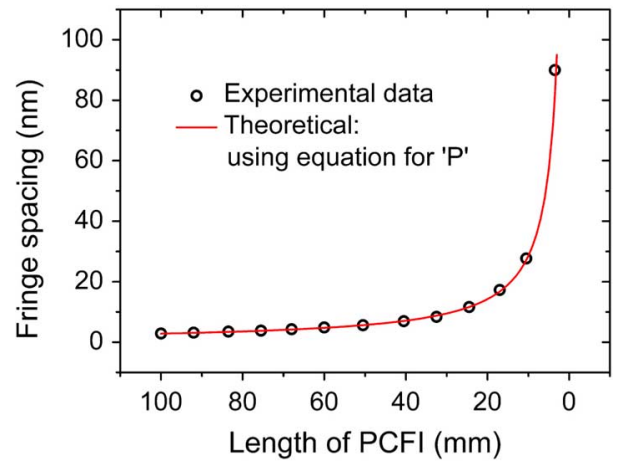

Fig. 4. The fringe spacing as a function of length of PCF observed for a reflection type interferometer.

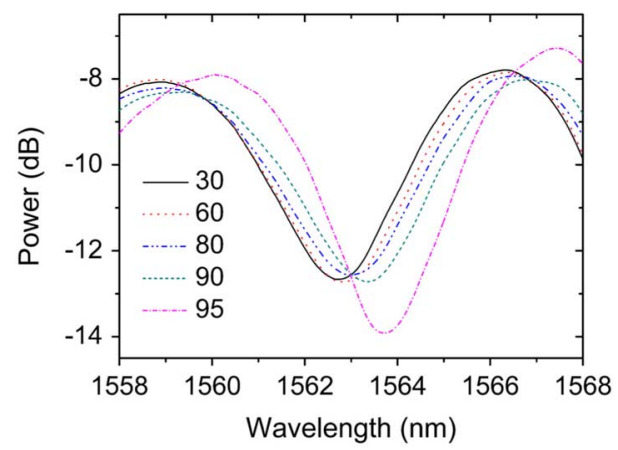

Fig. 5. Reflection spectrum of a $40.5 \mathrm{~mm}$ long PCFI at different humidity values.

dependence of the intermodal interference [20]. Fig. 4 shows the measured fringe spacing or periods of the fabricated PCFIs as a function of length of PCF. The measured periods agree well with the expected ones for a two-mode interferometer which is given by the expression $\mathrm{P} \approx \lambda^{2} /(2 \Delta n L)$. The value of $\Delta \mathrm{n}$ calculated based on the experimental data is $\sim 4.2 \times 10^{-3}$.

In order to study the influence of humidity/dew on the PCFI, it was decided to limit the PCFI length used to $42 \mathrm{~mm}$ or less, to suit the size of the available TEC used for temperature control. Initially the humidity response of the device is studied at a temperature $\left(25^{\circ} \mathrm{C}\right)$ and at normal atmospheric pressure by placing it in a controlled environmental chamber. Fig. 5 shows the changes in the reflection spectrum with respect to ambient 


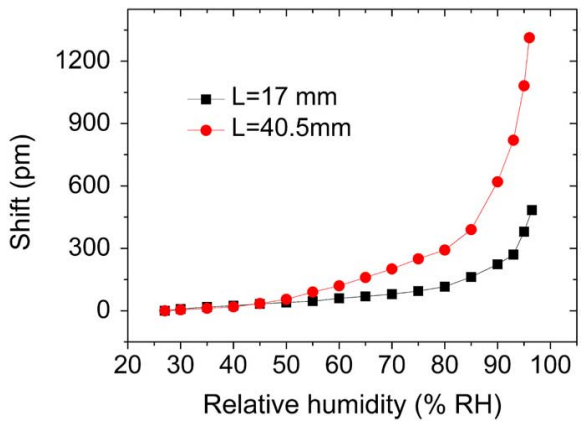

Fig. 6. Interference peak shift of the photonics crystal fiber interferometers with $\mathrm{L}=40.5 \mathrm{~mm}$ and $17 \mathrm{~mm}$ with respect to relative humidity.

humidity for a device with $\mathrm{L}=40.5 \mathrm{~mm}$. The change in the adsorption with respect to ambient humidity changes the effective refractive index of the cladding mode $\left(n_{\mathrm{cl}}\right)$. The resulting phase change in turn results in a shift of the interference pattern. The curves in Fig. 5 show the position of a zoomed section of the device spectrum at relative humidity values of $30,60,80$ and 90 $\%$ RH. When humidity increases the interference pattern shifts to longer wavelengths and this shift is more significant at higher humidity values. To study the effect of reducing the length of the PCFI a second PCFI was fabricated with a shorter length of $17 \mathrm{~mm}$. Fig. 6 shows the peak shift of the interferometer with respect to humidity obtained for two devices with $\mathrm{L}=17 \mathrm{~mm}$ and $40.5 \mathrm{~mm}$.

It is observed from the Fig. 6 that the sensitivity of the device to humidity decreases as the length of the device decreases. This is due to the fact that for a small device the interaction length available for interaction between the cladding mode with the adsorbed water vapor is less so the acquired phase difference between the interfering modes will be smaller. Hence, the sensitivity to humidity change is less for a device with a smaller length of PCF. To study the dew response of the PCF interferometer it is placed on a thermoelectric cooler (TEC) as shown in Fig. 2. The temperature of the TEC element is controlled by a temperature controller. A thermistor is used to provide temperature feedback to the controller from the TEC element. An additional handheld thermometer is used to confirm the temperature on the TEC surface. The entire setup is placed inside a controlled environmental chamber. The inside relative humidity and the temperature of the chamber can be controlled with an accuracy of $\pm 2 \% \mathrm{RH}$ and $\pm 1{ }^{\circ} \mathrm{C}$ respectively. For the purpose of this experiment the ambient temperature inside the chamber is fixed at $25^{\circ} \mathrm{C}$.

Since the PCF is composed of only fused silica, it is expected to have minimal thermal sensitivity. To determine the temperature dependence of the device the interference peak shift is observed while varying the temperature of the device from $25^{\circ} \mathrm{C}$ to $60^{\circ} \mathrm{C}$. The ambient humidity during the study was set to $40 \% \mathrm{RH}$. When the temperature is increased from $25^{\circ} \mathrm{C}$ to $60^{\circ} \mathrm{C}$ the interference peak is shifted slightly to higher wavelengths. Fig. 7 shows this temperature dependence for two devices with $\mathrm{L}=17 \mathrm{~mm}$ and $40.5 \mathrm{~mm}$. As expected the thermal sensitivity of the PCFI is very low and is further reduced for a device with the shorter length of PCF. The thermal sensitivity obtained in

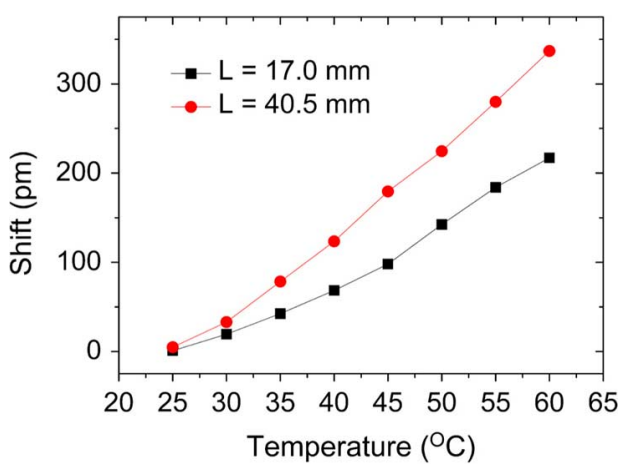

Fig. 7. Interference peak shift with respect to temperature for interferometers with PCF lengths $\mathrm{L}=40.5 \mathrm{~mm}$ and $17 \mathrm{~mm}$.

the experiment for a device with $\mathrm{L}=40.5 \mathrm{~mm}$ is $9.5 \mathrm{pm} /{ }^{\circ} \mathrm{C}$ and that for $\mathrm{L}=17 \mathrm{~mm}$ is $6.2 \mathrm{pm} /{ }^{\circ} \mathrm{C}$.

The dew sensing experiments are carried out at an ambient temperature of $25^{\circ} \mathrm{C}$ and at normal atmospheric pressure. To study the dew response of the device the temperature of the PCFI is decreased from ambient temperature $\left(25^{\circ} \mathrm{C}\right)$ to the dew point temperature at a fixed ambient relative humidity. It is found that the position of the interference peaks shifted to longer wavelengths with a decrease in temperature. This shift is similar to the humidity response of the PCFI as shown in Figs. 5 and 6 . This occurs because the relative humidity inside the microholes and close to the PCFI increases with a decrease in temperature and causes a shift. At or below the dew point temperature $(100 \% \mathrm{RH})$ water vapor condensation occurs, the condensed water vapor on the outer surface of the PCF also contribute to the change in the effective RI of the cladding mode, which results in a large spectral shift.

The spectra of two interferometers at room temperature and at the dew point temperature for devices fabricated with lengths $40.5 \mathrm{~mm}$ and $3.5 \mathrm{~mm}$ are shown in Fig. 8(a) \& (b). The lengths selected are practically the largest and the smallest PCF lengths that can be studied using our experimental setup. The ambient humidity during this study was set at $60 \%$ RH. From the Fig. 8 it is clear that relative to the period of the interferometer the shift will be larger for a longer PCFI due to a longer interaction length available for the interference between the cladding mode and the adsorbed water vapor. Hence, the sensitivity to water vapor content and thus dew point temperature is less for a device with a shorter length of PCF.

It is important to note that due to the large fringe spacing it is difficult to measure the peak shift accurately for a short PCFI, therefore the comparison of sensitivities for PCFIs with different lengths is not straightforward. It should be noted that even a PCFI with a small length $(3.5 \mathrm{~mm}$, fringe spacing $\sim 90 \mathrm{~nm})$ when exposed to dew point temperature for a relatively long time i.e., several minutes will result in a measurable fringe shift as shown in Fig. 8(b). This is because an increasingly thicker adsorbed water layer is formed on the silica surfaces of the PCF as time progresses. Thus, compared to $3.5 \mathrm{~mm}$ device the $\sim 40.5 \mathrm{~mm}$ device is preferable for achieving a fast response time (in the order of seconds), but when a compact length is the main requirement a shorter PCFI also can be used as a dew sensor with a reduced measurement speed. 


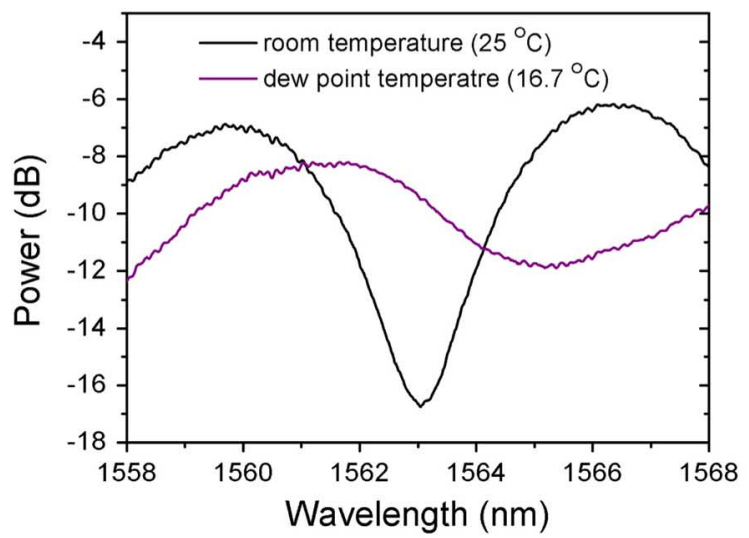

(a)

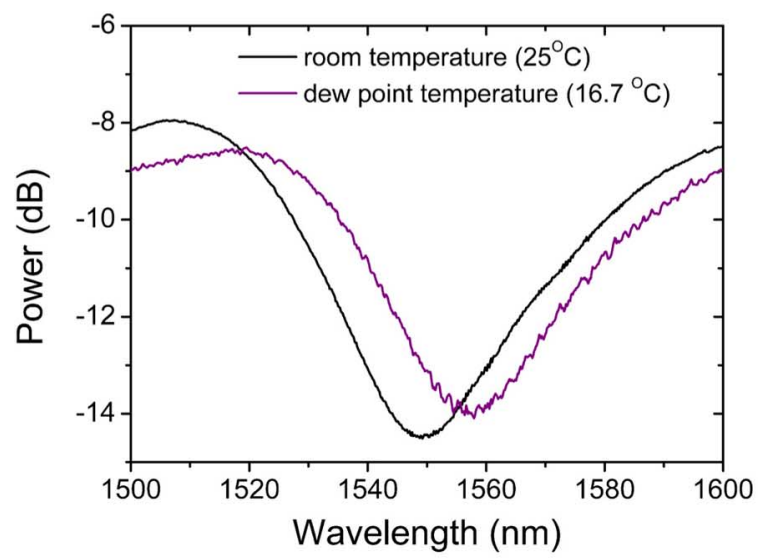

(b)

Fig. 8. (a) Interference spectra for a device with length $40.5 \mathrm{~mm}$ at room temperature and at dew point temperature. (b) Interference spectra for a device with length $3.5 \mathrm{~mm}$ at room temperature and at dew point temperature.

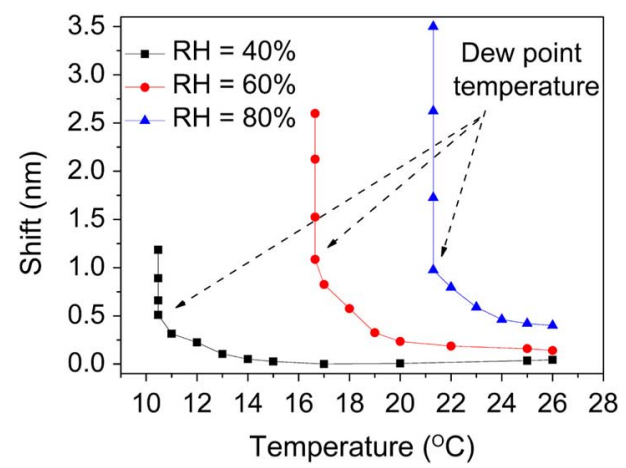

Fig. 9. Interference peak shift of PCFI with respect to temperature at three ambient humidity values of 40,60 and $80 \% \mathrm{RH}$.

To determine the dew sensing performance of a PCFI at different environmental conditions, we study the dew response of a PCFI with $\mathrm{L}=40.5 \mathrm{~mm}$ at three ambient humidity values of 40, 60 and $80 \%$ RH. At each humidity value the temperature of the PCFI is reduced from $26^{\circ} \mathrm{C}$ to the corresponding dew point temperature. The peak wavelength shift of the device is plotted against temperature in Fig. 9. The three curves represent the peak shift corresponding to the ambient relative humidity values of 40, 60 and $80 \% \mathrm{RH}$. The onset of the dew formation is characterized by a large shift of the interference peak which is clear in Fig. 9. The dew point temperature calculated by using (1) based on the corresponding ambient conditions is marked on each curve in Fig. 9. For all these three ambient humidity values the continuous spectral shift starts exactly at the dew point temperature which confirms the high dew point measurement accuracy (estimated as $\pm 0.1^{\circ} \mathrm{C}$ ) of the sensor.

It is observed that at or below the dew point temperature the interference peak shifts continuously with time. This is because an increasingly thicker adsorbed water layer is formed on the silica surface of the PCF microholes as time progresses. By bringing the temperature of the PCFI back to room temperature the interference peaks also shift back to their initial position. This shows the reversibility of the sensor. Because of the small size of the sensor head and the high sensitivity to adsorbed water vapor the demonstrated sensor response time is in seconds which is relatively fast compared to existing dew point hygrometers that take several minutes for a single measurement. The simple fabrication method, small size and the all-silica nature of the demonstrated sensor head suggest that with some simple additions such as attaching a TEC element with temperature feedback on to the PCFI, the combination can be used as a dew point hygrometer.

\section{CONCLUSION}

A PCFI sensor that operates in reflection mode is presented in this paper for dew detection. The fabrication of the sensor head is simple since it only involves cleaving and fusion splicing. A reversible interference peak shift is observed for the sensor. The measurement accuracy and the response time of the PCFI are better compared to the existing dew point hygrometers. It is also concluded that by attaching a TEC element with temperature feedback the proposed PCFI can be used as a miniature dew point hygrometer.

\section{REFERENCES}

[1] D. Camuffo and S. Valcher, "A dew point signaller for conservation of works of art," Environ. Monit. Assess, vol. 6, no. 2, pp. 165-170, 1986.

[2] A. Kumada, E. Takata, and M. Murata, "Frost and Dew Sensor," U.S. Patent 5000579, Mar. 19, 1991.

[3] R. S. Jachowicz, J. Weremczuk, D. Paczesny, and G. Tarapata, "A MEMS-based super fast dew point hygrometer-Construction and medical applications," Meas. Sci. Technol., vol. 20, no. 12, p. 124008 , 2009, (10pp).

[4] F. Pascal-Delannoy, B. Sorli, and A. Boyer, "Quartz crystal microbalance (QCM) used as humidity sensor," Sens. Actuators A, vol. 84, no. 3, pp. 285-291, Sep. 2000.

[5] M. Hoummady, C. Bonjour, J. Collin, F. Lardet-Vieudrin, and G. Martin, "Surface acoustic wave (SAW) dew point sensor: Application to dew point hygrometry," Sens. Actuators B, vol. 27, no. 1-3, pp. 315-317, Jun. 1995, Eurosensors VIII.

[6] P. R. Stoddart, J. B. Pearce, and A. P. Mazzolini, "Evanescently coupled dewpoint sensor based on a silicon waveguide," Sens. Actuators $A$, vol. 128 , pp. 225-229, 2006

[7] T. Numata, Y. Otani, and N. Umeda, "Optical dew sensor using surface plasmon resonance of periodic ag nanostructure," Jpn. J. Appl. Phys., vol. 45, no. 30, pp. L810-L813, 2006.

[8] P. Popov, S. Sainov, and M. Baeva, "Measurement of surface dew by optical sensor," Sens. Actuators A, vol. 51, no. 2-3, pp. 199-202, 1996.

[9] S. Hadjiloucas, J. Irvine, and D. A. Keating, "Feedback dew-point sensor utilizing optimally cut plastic optical fibres," Meas. Sci. Technol., vol. 11, no. 1, p. 1, 2000.

[10] O. Postolache, P. M. B. S. Girão, J. M. D. Pereira, and H. G. Ramos, "Dew point and relative-humidity smart measuring system," IEEE Trans. Instrum. Meas., vol. 55, no. 6, 2006. 
[11] F. Baldini, R. Falciai, A. A. Mencaglia, F. Senesi, D. Camuffo, A. D. Valle, and C. J. Bergsten, "Miniaturised optical fibre sensor for dew detection inside organ pipes," Journal of Sensors, vol. 2008, Article ID 321065 .

[12] S. M. Kostritskii, A. A. Dikevich, Yu. N. Korkishko, and V. A. Fedorov, "Dew point measurement technique utilizing fiber cut reflection," in Proc. SPIE, 2009, p. 7356, 73561K.

[13] J. Mathew, Y. Semenova, G. Rajan, and G. Farrell, "Photonic crystal fiber interferometer for dew detection," in Proc. SPIE, 2011, p. 7753, $77531 \mathrm{P}$.

[14] J. Villatoro, V. P. Minkovich, V. Pruneri, and G. Badenes, "Simple allmicrostructured-optical-fiber interferometer built via fusion splicing," Opt. Express, vol. 15, no. 4, pp. 1491-1496, 2007.

[15] J. Villatoro, M. P. Kreuzer, R. Jha, V. P. Minkovich, V. Finazzi, G. Badenes, and V. Pruneri, "Photonic crystal fiber interferometer for chemical vapor detection with high sensitivity," Opt. Express, vol. 17, no. 3, pp. 1447-1453, 2009.

[16] D. Barrera, J. Villatoro, V. P. Finazzi, G. A. Cardenas-Sevilla, V. P. Minkovich, S. Sales, and V. Pruneri, "Low-loss photonic crystal fiber interferometers for sensor networks," J. Lightwave Technol., vol. 28, no. 24 , pp. $3542-3547,2010$.

[17] J. Villatoro, V. Finazzi, G. Badenes, and V. Pruneri, "Highly sensitive sensors based on photonic crystal fiber modal interferometers," Journal of Sensors, vol. 2009, p. 11, 2009, Article ID 747803.

[18] H. Y. Choi, M. J. Kim, and B. H. Lee, "All-fiber Mach-Zehnder type interferometers formed in photonic crystal fiber," Opt. Express, vol. 15, no. 9, pp. 5711-5720, 2007.

[19] R. Jha, J. Villatoro, and G. Badenes, "Ultrastable in reflection photonic crystal fiber modal interferometer for accurate refractive index sensing," Appl. Phys. Lett., vol. 93, no. 19, p. 3, 2008, Article ID 191106.

[20] W. J. Bock, T. A. Eftimov, P. Mikulic, and J. Chen, "An inline corecladding intermodal interferometer using a photonic crystal fiber," $J$. Lightwave Technol., vol. 27, no. 17, pp. 3933-3939, 2009.

[21] G. A. Cárdenas-Sevilla, V. Finazzi, J. Villatoro, and V. Pruneri, "Photonic crystal fiber sensor array based on modes overlapping," Opt. Express, vol. 19, pp. 7596-7602, 2011.

[22] J. Mathew, Y. Semenova, G. Rajan, and G. Farrell, "Humidity sensor based on photonic crystal fibre interferometer," Electron. Lett., vol. 46, no. 19 , pp. 1341-1343, 2010

[23] D. Káčik, I. Turek, I. Martinček, J. Canning, N. Issa, and K. Lyytikäinen, "Intermodal interference in a photonic crystal fibre," Opt. Express, vol. 12, pp. 3465-3470, 2004.

[24] H. P. Uranus, "Theoretical study on the multimodeness of a commercial endlessly single-mode PCF," Opt. Commun., vol. 283, no. 23, pp. 4649-4654, 2010.

[25] B. A. David and H. K. Seong, "Evolution of the adsorbed water layer structure on silicon oxide at room temperature," J. Phys. Chem. B, vol. 109 , no. 35 , pp. 16760-16763, 2005.
[26] K. Tiefenthaler and W. Lukosz, "Grating couplers as integrated optical humidity and gas sensors," Thin Solid Films, vol. 126, pp. 205-211, 1985.

Mr. Jinesh Mathew received his M.Sc. degree in electronic science and the M.Phil. degree in photonics from Cochin University of Science and Technology, India. In 2008, he joined the Photonics Research Center, Dublin Institute of Technology, Dublin, Ireland, as a postgraduate researcher. His current research interests include biological and environmental parameters sensing using optical fiber. Jinesh Mathew has more than five years of research experience in the area of fiber optic sensors and has published over 18 journal and conference papers in this area.

Dr. Yuliya Semenova is a graduate of Lviv Polytechnic National University (Ukraine) of 1992. She received her Ph.D. Degree in physics of liquid crystals from the Ukrainian Academy of Sciences in 1999. Between 1997 and 2001 she worked as a researcher at the faculty of Electrophysics at the Lviv Polytechnic National University. Since 2001 she has been with the School of Electronic and Communications Engineering at Dublin Institute of Technology. She is a lecturer and senior researcher in the Photonics Research Center. Her research interests include liquid crystals, photonics and fiber optic sensing. She has published over 100 journal and conference papers.

Prof. Gerald Farrell is the Principal Investigator and Director of the Photonics Research Center at the Dublin Institute of Technology. He graduated with an honours degree in electronic engineering from University College Dublin in 1979 and spent a number of years as a communications systems design engineer developing optical fiber transmission systems before joining the DIT. He has a Ph.D. from Trinity College Dublin for research in all-optical synchronization using self-pulsating laser diodes. He is also Head of School of the School of Electronic and Communications Engineering at the DIT since 2001. His current research interests lie in several areas of optical sensing including FBG interrogation systems; the modeling and applications of fiber bend loss to optical sensing; SMS and other fiber structures for sensing applications; PCF sensors for environmental sensing and for sensing strain in composite materials and medical devices, $L C$ infiltrated PCF sensors; and micro-fiber and nanowire sensors for bio-sensing. Prof. Farrell has over 180 publications in the area of photonics and holds several patents. Between 1997 and 2003 he was a director of the startup company PX Instrument Technology, focusing on optical fiber system test and measurement systems. 